\title{
$30 \mathrm{GHz}$ monitoring of broad absorption line (BAL) quasars
}

\section{Maciej Ceglowski ${ }^{*}$}

Torun Centre for Astronomy, Gagarina 11, 87-100 Torun, Poland

E-mail: ceglowskieastri.uni.torun.pl

\section{Magdalena Kunert-Bajraszewska}

Torun Centre for Astronomy, Gagarina 11, 87-100 Torun, Poland

E-mail: magda@astro.uni.torun.pl

\section{Bogna Pazderska}

Torun Centre for Astronomy, Gagarina 11, 87-100 Torun, Poland

E-mail: bogna@astro.uni.torun.pl

\section{Marcin Gawronski}

Torun Centre for Astronomy, Gagarina 11, 87-100 Torun, Poland

E-mail: motylekeastro.uni.torun.pl

Broad absorption line (BAL) quasars have been studied for over thirty years. Yet it is still unclear why and when we observe broad absorption lines in quasars. Is this phenomenon caused by geometry or is it connected with the evolution process? Variability of the BAL quasars, if present, can give us information about their orientation, namely it can indicate whether they are oriented more pole-on. Using the Torun 32-metre dish equipped with the One Centimetre Receiver Array (OCRA) we have started a monitoring campaign of a sample of compact radio-loud BAL quasars. This $30 \mathrm{GHz}$ variability monitoring program supplements the high-resolution interferometric observations of these objects we have carried out with the EVN and VLBA.

11th European VLBI Network Symposium \& Users Meeting

9-12 October 2012

Bordeaux (France)

\footnotetext{
*Speaker.
} 


\section{Introduction}

Broad absorption lines (BALs) are seen in a small (15\%) fraction of both the radio-quiet and radio-loud quasar populations [10], according to the traditional BALQSO definition of [18]. They are probably caused by outflow of gas with high velocities and are part of the accretion process. The presence of BALs is probably a geometrical effect [5] but it can be also connected with quasar evolution [7]. Theoretical models suggest that BALs are seen at high inclination angles, which means that the outflows from accretion disks are present near the equatorial plane. However, there are also observational evidences indicating the existence of polar outflows from the inner regions of a thin disk $[19,6]$. This means that either there is not a simple orientation model which can explain all the features observed in BALQSOs and/or the evolution scenario should be taken into account.

The evolution scenario which emerged with the discovery of radio-loud BALQSOs suggests that every quasar has a BAL phase at the beginning of its lifetime [1]. Recent analysis of the spectral shape, variability and polarization properties of some of the compact radio-loud BALQSOs [15] indicates that indeed they are similar to young compact steep spectrum (CSS) and gigahertz peaked spectrum (GPS) objects.

The VLBI technique is still the best way to learn about morphologies and orientation of BAL quasars. However, only a very small fraction of compact BAL quasars have been observed with VLBI so far $[9,14,15,11,4,13,3,8]$. About half of the observed sources have unresolved structures which prevents us from directly estimating their orientation from morphology, but the steepness of their radio spectrum can give us an idea about their orientation. Additionally, BAL quasars with polar rather than equatorial outflows have likely been identified via short timescale variability [19].

A few years ago we started high-resolution EVN and VLBA observations of selected samples of compact radio-loud broad absorption line quasars to study their morphology [12]. Last year we have also initiated a flux variability monitoring campaign of these samples with the One Centimetre Receiver Array (OCRA) prototype system mounted on the Torun $32 \mathrm{~m}$ radiotelescope.

\section{OCRA receiver}

The OCRA project aims to construct a one-hundred-beam receiver system, operating in a frequency band centred on $30 \mathrm{GHz}$ [2]. This system is to be installed on the $32 \mathrm{~m}$ parabolic antenna at Torun Observatory. The antenna is ideal for high-frequency multi-beam work as it has very accurate reflector panels and paraboloid/hyperboloid (non-shaped) optics. Having 100 beams would allow all-sky surveys to be carried out much quicker than is currently possible. Currently there are two ongoing projects, one designated OCRA-p and the other OCRA-F. They are being used to develop the technology needed for the full OCRA system. OCRA-p is a two-beam prototype receiver, based on the design of the $30 \mathrm{GHz}$ Planck Low Frequency Instrument, and OCRA-F is an 8-beam receiver using the MMICs technology [16]. Our monitoring of BAL quasars is carried out with OCRA-p. The sensitivity and stability of the receiver allow us to observe sources as weak as $10 \mathrm{mJy}$, with improvements still going on. 


\section{Sample and observations}

We have cross-identified the FIRST survey with a catalog of optically-selected BAL quasars from SDSS/DR3 [17] with the following selection criteria: a) unresolved, isolated sources i.e more compact than the FIRST beam (5.4 arcsec), b) sources surrounded by an empty field within a radius of 1 arcmin, c) sources with a flux density $S_{1.4 \mathrm{GHz}}>20 \mathrm{mJy}$. The final sample consist of 26 compact radio-loud BAL quasars.

We have already observed part of the sample with the VLBA at 5 and $8.4 \mathrm{GHz}$ in polarization mode. The remaining objects of the sample are currently being observed with the EVN at $5 \mathrm{GHz}$. Most of the sources that have been observed so far reveal core-jet structures and show fainter emission from the jet compared to the core. This morphology suggests intermediate orientation between polar and equatorial geometries and supports the orientation scenario. For some sources the flux densities of the observed compact structures account only for up to $25 \%$ of the total flux density at $5 \mathrm{GHz}$. This suggests that these sources comprise low brightness extended structures and may be older and bigger than GPS/CSS objects [12].

The $30 \mathrm{GHz}$ monitoring of the whole sample is being carried out using OCRA-p in "on-off" mode. Each source is observed once per month and each flux measurement is done twice. We have divided our sample into two groups of objects based on their $1.4 \mathrm{GHz}$ flux density. The $S_{1}$ group consists of the 16 brighter sources with flux densities $S_{1.4 \mathrm{GHz}}>150 \mathrm{mJy}$. The second group $S_{2}$ consists of 10 much weaker objects with flux densities $S_{1.4 \mathrm{GHz}}>20 \mathrm{mJy} .14$ out of the 16 sources from the $S_{1}$ group have been detected at $30 \mathrm{GHz}$ so far,and their flux density values range from $9.5 \mathrm{mJy}$ to $270 \mathrm{mJy}$. Their spectral indices between $1.4 \mathrm{GHz}$ and $5 \mathrm{GHz}$ range from -0.33 to 0.9 and get steeper between $5 \mathrm{GHz}$ and $30 \mathrm{GHz}$. Sources belonging to the $S_{2}$ group have flux density that is more than 3 times weaker and only 3 of them have been detected at $30 \mathrm{GHz}$ so far. Weather conditions play an important role as far as data quality is concerned. However, with the monitoring going on, this limitation will be overcome as more data are acquired.

By carrying out this project we intend to address several important issues, one of which is to find out the BAL quasar orientation. Short timescale radio flux variations of BALQSOs may help to confirm the previous findings that viewing angles are within $35^{\circ}$ of the jet axis [6]. From a preliminary analysis of our high-resolution VLBI observations, supplemented with those found in the litterature, we suspect that flux and polarisation variability is present in some of them. Our second goal is to obtain the spectral energy distribution (SED) of these BALQSOs. This will allow us to study their spectral shape, namely its steepness, and check the potential presence of low brightness extended structures.

\section{Acknowledgements}

This work was supported by the Polish Ministry of Science and Higher Education under grant UMO2011/01/D/ST9/00378. We gratefully acknowledge the financial support of the Royal Society Paul Instrument Fund which allowed us to build the $30 \mathrm{GHz}$ OCRA receiver. We are also grateful to the Polish Ministry of Science and Higher Education which provided support for operating the receiver on the Torun 32-m telescope. 


\section{References}

[1] Becker, R. H., White, R. L., Gregg, M. D., Brotherton, M. S., Laurent-Muehleisen, S. A., \& Arav, N., 2000, ApJ, 538, 72

[2] Browne, I. W., Mao, S., Wilkinson, P. N., Kus, A. J., Marecki, A., \& Birkinshaw, M., 2000, Proc. SPIE, 4015, 299

[3] Bruni, G., Dallacasa, D., Mack, K.-H., et al., 2013, A\&A, 554, 94

[4] Doi, A., et al., 2009, PASJ, 61, 1389

[5] Elvis, M., 2000, ApJ, 545, 63

[6] Ghosh, K. K., \& Punsly, B., 2007, 661, L139

[7] Gregg, M. D., Becker, R. H., Brotherton, M. S., Laurent-Muehleisen, S. A., Lacy, M., \& White, R. L., 2000, ApJ, 544, 142

[8] Hayashi, T. J., Doi, A., \& Nagai, H., 2013, ApJ, 772, 4

[9] Jiang, D. R., \& Wang, T. G., 2003, A\&A, 397, L13

[10] Knigge, C., Scaringi, S., Goad, M. R., \& Cottis, C. E., 2008, MNRAS, 386, 1426

[11] Kunert-Bajraszewska, M., Siemiginowska, A., Katarzyński, K., \& Janiuk, A., 2009, ApJ, 705, 1356

[12] Gawroński, M. P., \& Kunert-Bajraszewska, M., 2011, PoS (10th EVN Symposium)037

[13] Kunert-Bajraszewska, M., Janiuk, A., Gawroński, M. P., \& Siemiginowska, A., 2010, ApJ, 718, 1345

[14] Liu, Y., Jiang, D. R., Wang, T. G., \& Xie, F. G., 2008, MNRAS, 391, 246

[15] Montenegro-Montes, F. M., Mack, K.-H., Vigotti, M., et al., 2008, MNRAS, 388, 1853

[16] Pengelly, R. S., 1975, Electronics Letters, 11, 58

[17] Trump, J. R., Hall, P. B., Reichard, T. A., Richards, G. T., et al., 2006, ApJS, 165, 1

[18] Weymann, R. J., Morris, S. L., Foltz, C. B., \& Hewett, P. C., 1991, ApJ, 373, 23

[19] Zhou, H., Wang, T., Wang, H., Wang, J., Yuan,W., \& Lu, Y., 2006, ApJ, 639, 716 